\title{
Cored Rutherford Cables for the GSI Fast Ramping Synchrotron
}

\author{
M. N. Wilson, Member, IEEE, A. K. Ghosh, B. ten Haken, W. V. Hassenzahl, Member, IEEE, J. Kaugerts, G. Moritz, \\ C. Muehle, A. den Ouden, R. Soika, P. Wanderer, and W. A. J. Wessel
}

\begin{abstract}
The new heavy ion synchrotron facility proposed by GSI will have two superconducting magnet rings in the same tunnel, with rigidities of $200 \mathrm{~T} \cdot \mathrm{m}$ and $100 \mathrm{~T} \cdot \mathrm{m}$. Fast ramp times are needed, which can cause significant problems for the magnets, particularly in the areas of ac loss and field distortion. This paper discusses the $200 \mathrm{~T} \cdot \mathrm{m}$ ring, which will use $\operatorname{Cos} \theta$ magnets based on the RHIC dipole design. We discuss the reasons for choosing Rutherford cable with a resistive core and report loss measurements carried out on cable samples. These measurements are compared with theoretical calculations using measured values of inter-strand resistance. Reasonably good agreement is found, but there are indications of nonuniformity in the adjacent resistance $R_{a}$. Using these measured parameters, losses and temperature rise are calculated for a RHIC dipole in the operating cycle of the accelerator. A novel insulation scheme designed to promote efficient cooling is described.
\end{abstract}

Index Terms-AC loss, dipole magnet, field error, Rutherford cable, superconducting synchrotron.

\section{INTRODUCTION}

G SI IS planning a new heavy ion accelerator consisting of two superconducting synchrotron rings placed one above the other in the same tunnel [1], and ramping with a rise time of a few seconds. The lower ring, having a magnetic rigidity of 100 $\mathrm{T} \cdot \mathrm{m}$, will use magnets based on the Nuclotron design [2] and the upper $200 \mathrm{~T} \cdot \mathrm{m}$ ring will use magnets based on the RHIC design [3]. Here we consider the cable for the RHIC dipoles, which use a single layer $\operatorname{Cos} \theta$ winding. To reach the high average intensities required by the project, it will be necessary to ramp the magnets at a rate of $\sim 1 \mathrm{~T} / \mathrm{s}$, with the following operating cycle:

inject at $0.4 \mathrm{~T}$

ramp up to $4 \mathrm{~T}$ at $1 \mathrm{~T} / \mathrm{s}$

extract at $4 \mathrm{~T}$ for $5 \mathrm{~s}$

ramp down at $1 \mathrm{~T} / \mathrm{s}$.

Manuscript received August 5, 2002. This work was supported in part by the U.S. Department of Energy under Contract DE-AC02-98CH10886.

M. N. Wilson is with Brook House, Abingdon, OX14 3AY, U.K. (e-mail: m-wilson@clara.co.uk).

A. K. Ghosh, R. Soika, and P. Wanderer are with the Brookhaven National Laboratory, Upton, NY 11973 USA (e-mail: aghosh@ bnl.gov; soika@ bnl.gov; wanderer@bnl.gov).

B. ten Haken, A. den Ouden, and W. E. J. Wessel are with the Low Temperature Division, University of Twente, 7500 AE Enschede, Netherlands (e-mail: b.tenhaken@tn.utwente.nl; A.denOuden@tn.utwente.nl; w.a.j.wessel@tn.utwente.nl).

W.V. Hassenzahl is with Advanced Energy Analysis, Piedmont, CA 94611 USA (e-mail: advenergy1@aol.com).

J. Kaugerts, G. Moritz, and C. Muehle are with GSI, Abteilung BTE, D-64291, Darmstadt, Germany (e-mail: G.Moritz@gsi.de; j.kaugerts@gsi.de; C.Muehle@gsi.de).

Digital Object Identifier 10.1109/TASC.2003.812864
To date, superconducting accelerators with high field magnets have all worked at relatively slow ramp rates and have been able to use Rutherford cable without incurring too much ac loss. At $1 \mathrm{~T} / \mathrm{s}$ however, coupling between the strands of a conventional Rutherford cable would produce high ac losses and unacceptable field distortion. Coupling may be reduced by increasing the resistance between strands in the cable, but there are reasons to believe that this can impede current sharing and thereby make the magnet more susceptible to quenching at high ramp rates. Because coupling in Rutherford cables is very anisotropic, it may be reduced greatly by increasing the crossover resistance via a core foil, while still leaving a low resistance in the other direction. Cables with core foils were first tried more than 20 years ago [4] and have been the subject ongoing research [5]. We are hopeful that cores will reduce the losses without affecting current sharing.

We first discuss the general question of losses and current sharing, leading to our choice of a cored cable. Loss measurements on prototype cables are described and compared with theoretical predictions based on Ohmic measurements of interstrand resistance. This formulation is then used to predict the loss and temperature rise in an operational dipole magnet. A novel insulation scheme with cooling holes in the Kapton wrap is described.

\section{CURRENT Sharing AND LOSSES}

Coupling currents in a Rutherford cable flow between the strands via two kinds of inter-strand resistance: the crossover resistance $R_{c}$ and adjacent resistance $R_{a}$ as sketched in Fig. 1 .

We can reduce coupling losses by increasing $R_{c}$ and $R_{a}$ - so why not simply make the inter-strand resistance as large as is needed to reduce losses to the required level? The main reason for not doing so is that a high inter-strand resistance seems to degrade the quench current at high ramp rates; we suggest two possible mechanisms for this. Firstly, it has been shown experimentally and theoretically [6] that partially soldered cables (low $R_{c}$ and $R_{a}$ ) have a minimum quench energy MQE at high currents which is $\sim 5 \times$ higher than the same cable unsoldered (high $R_{c}$ and $R_{a}$ ). Thus a low inter-strand resistance seems to bring greater stability against random disturbances (e.g., wire movement) in the magnet.

Secondly there is the phenomenon of ramp rate induced quenching, found in some fusion magnets and investigated in detail during the development of magnets for the SSC High Energy Booster [7]. As shown in Fig. 2, these magnets went to their full critical current at slow ramp rates, but quenched early when the current was ramped up quickly. Two types of 


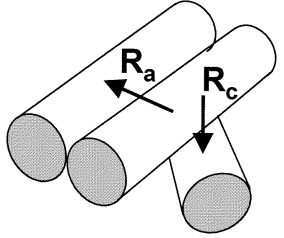

Fig. 1. Crossover and adjacent resistances between strands in a cable (note that $R_{a}$ is defined to be over the same length of wire that $R_{c}$ occupies).

behavior were identified. Type A magnets showed no reduction in quench current until a rate of $\sim 25 \mathrm{~A} / \mathrm{s}$ was reached, after which the quench current decreased more or less linearly with ramp rate. Type $B$ magnets however suffered a rapid drop in quench current at quite small ramp rates. In general, it was found that magnets with small inter-strand resistance were Type A and those with large inter-strand resistance were type B. For example the Type A magnet in Fig. 2 had $R_{c}=9 \mu \Omega$ and Type B had $R_{c}=80 \mu \Omega$.

A plausible explanation of this behavior is that Type A quenching is caused by heating due to the ac losses arising from strong inter-strand coupling. Type B quenching is thought to be caused by nonuniform current distribution between the strands. It is well known that the current does not divide equally between the strands in accelerator magnets, as evidenced by the periodic variation of field along the length of the magnet, which has the same periodicity as the cable twist [8]. During ramping, if one strand has more current than its neighbors, it will reach critical current early and be driven into the flux-flow resistance region. When there is a good electrical contact to neighboring strands, the flux-flow voltage will drive some current into those strands and produce a more uniform distribution. If however the electrical contact is not good, the "victim" strand will have to go much further into the flux flow region in order to generate sufficient voltage to transfer the current. This extra flux-flow resistance may be enough to trigger a quench.

As discussed later, we are fairly confident that our new system of cooling holes will allow us to control Type A quenching, but we are nervous about Type B quenching. In the absence of a definite theory, we have therefore decided to make the contact resistance high enough to control the losses, but no higher than necessary. To quantify how high, we need to look at the three types of inter-strand coupling in Rutherford cable.

\section{A. Cable Coupling Via $R_{c}$ in Transverse Field}

$$
P_{t c}=\frac{1}{120} \frac{\dot{B}_{t}^{2}}{R_{c}} \frac{c}{b} p N(N-1)
$$

where $P_{t c}$ is the coupling loss per unit volume of cable, $p$ is the cable twist pitch, $\dot{B}_{t}$ is the rate of change of field transverse to the broad face of the cable, $N$ is the number of strands, $c$ is the half width of the cable and $b$ is its half thickness.

\section{B. Cable Coupling Via $R_{a}$ in Transverse Field}

$$
P_{t a}=\frac{1}{24} \frac{\dot{B}_{t}^{2}}{R_{a}}(N-1) \frac{p}{\operatorname{Cos}^{2} \theta}
$$

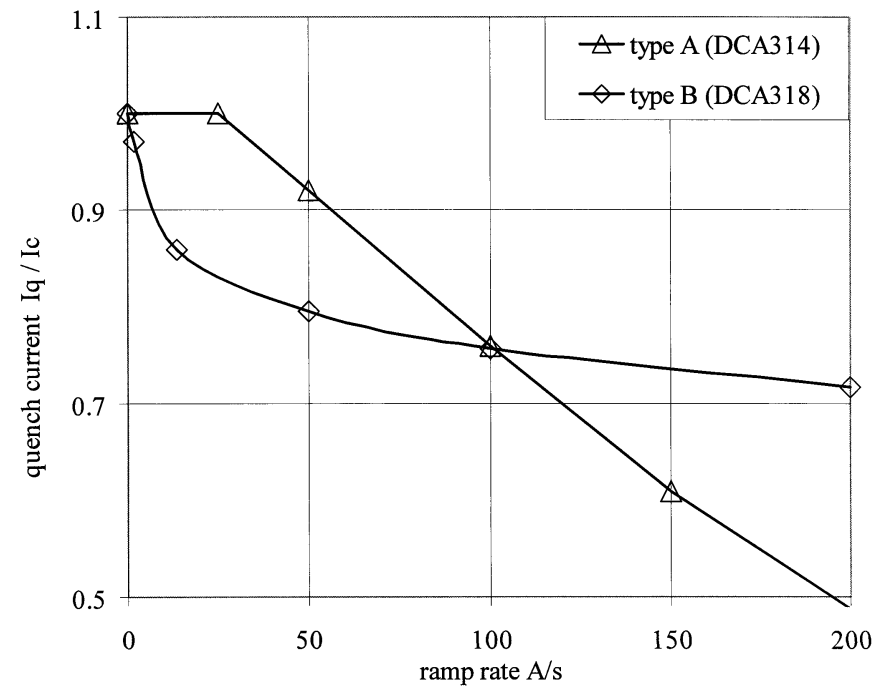

Fig. 2. Quench current of SSC magnets as a function of ramp rate [5].

where $\theta$ is the slope angle of the wire relative to the cable length $(\operatorname{Cos} \theta \sim 1)$.

\section{Cable Coupling Via $R_{a}$ in Parallel Field}

$$
P_{p a}=\frac{1}{32} \frac{\dot{B}_{p}^{2}}{R_{a}}(N-1) p \frac{b^{2}}{c^{2}} \frac{1}{\operatorname{Cos}^{2} \theta}
$$

where $\dot{B}_{p}$ is the rate of change of field parallel to the broad face of the cable. Because $c$ is always much greater than $b$, it may be seen immediately that the loss in transverse field is much greater than in parallel field. From (1) and (2) we see that the ratio:

$$
\frac{P_{t c}}{P_{t a}}=\frac{R_{a}}{R_{c}}\left\{\frac{N}{5} \frac{c}{b} \cos ^{2} \theta\right\} .
$$

A typical value for the factor in brackets is $\sim 50$, which means that a given crossover resistance causes 50 times more loss than the same adjacent resistance. It follows that we can make $R_{a} \sim$ $R_{c} / 50$ without increasing the loss too much. It is this inherent anisotropy in the loss mechanism that is the reason for choosing cored cables.

\section{SAMPLES AND LOSS MEASUREMENT}

Figs. 3 and 4 show cross sections of our prototype cable with a $25 \mu \mathrm{m}$ stainless steel core in its center. In other respects it is the same as the RHIC dipole cable [3].

Table I lists the common parameters of the cables tested, very similar to the RHIC dipole cables. The wires, manufactured by Oxford Superconducting Technology, were coated with "Staybrite" silver tin alloy and cabled by New England Electric.

Cables were made with different cores; Table II lists the parameters. Our preferred core material is stainless steel, but a brass core was also tested to get a result with lower $R_{c}$. Early attempts to use an anodized titanium foil were abandoned because the foil broke up during cabling. Even the stainless steel suffered some punching through near the (narrow) inner edge of the cable. To avoid this problem, we made cable 003E with two stainless steel cores. Adding the cores increased the half 


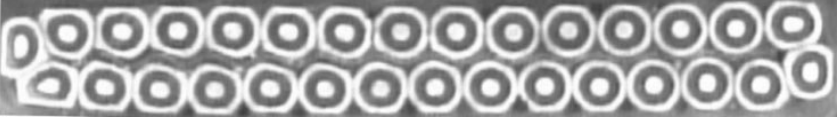

Fig. 3. Cross section of a cored cable.

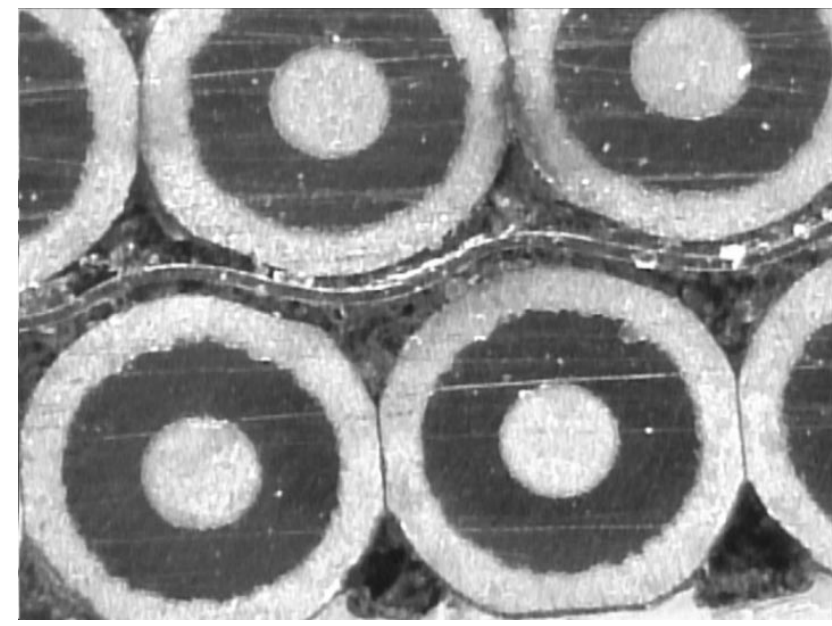

Fig. 4. Local region showing the stainless steel core foil.

TABLE I

COMmon PARAmeters OF THE CABLES Tested

\begin{tabular}{lll}
\hline cable twist pitch & $p$ & $74 \mathrm{~mm}$ \\
cable mean half thickness & $b$ & $0.583 \mathrm{~mm}$ \\
cable half width & $c$ & $4.93 \mathrm{~mm}$ \\
wire diameter & $d_{w}$ & $0.648 \mathrm{~mm}$ \\
wire copper / NbTi ratio & $m$ & 2.25 \\
filament diameter & $d_{f}$ & $6 \mu \mathrm{m}$ \\
\hline
\end{tabular}

TABLE II

VARYING PARAMETERS OF THE CABLES TESTED

\begin{tabular}{lll}
\hline cable & core & wire twist pitch \\
\hline $003 \mathrm{~B}$ & $1 \times 25 \mu \mathrm{m}$ stainless & $4 \mathrm{~mm}$ \\
$003 \mathrm{E}$ & $2 \times 25 \mu \mathrm{m}$ stainless & $6 \mathrm{~mm}$ \\
$003 \mathrm{~F}$ & $1 \times 50 \mu \mathrm{m}$ brass & $6 \mathrm{~mm}$ \\
\hline
\end{tabular}

thickness by 4 to $7 \mu \mathrm{m}$ above the value shown in Table I, so the compaction was somewhat greater than RHIC cable.

Losses were measured at the University of Twente. The sample, comprising 10 pieces of cable, each $375 \mathrm{~mm}$ long, was first compressed to $60 \mathrm{Mpa}$ and heated to $225 \mathrm{C}$ in a cycle which simulates the RHIC cure cycle for the Kapton insulation. The sample was then unloaded, transferred to the measurement sample holder and re-compressed to $60 \mathrm{Mpa}$. Losses were measured calorimetrically [9] in a superconducting dipole magnet, generating a sinusoidal oscillating field of $\pm 0.3 \mathrm{~T}$ at frequencies up to $250 \mathrm{mHz}$. By rotating the sample, the field could be oriented parallel or transverse to the face of the cable. Losses were measured in terms of gas flow, which was calibrated over the measurement range to an accuracy of $2 \%$. After a settling time of 10 minutes, each flow measurement was made for 5 minutes. Fig. 5 shows a typical result of loss per cycle versus frequency.

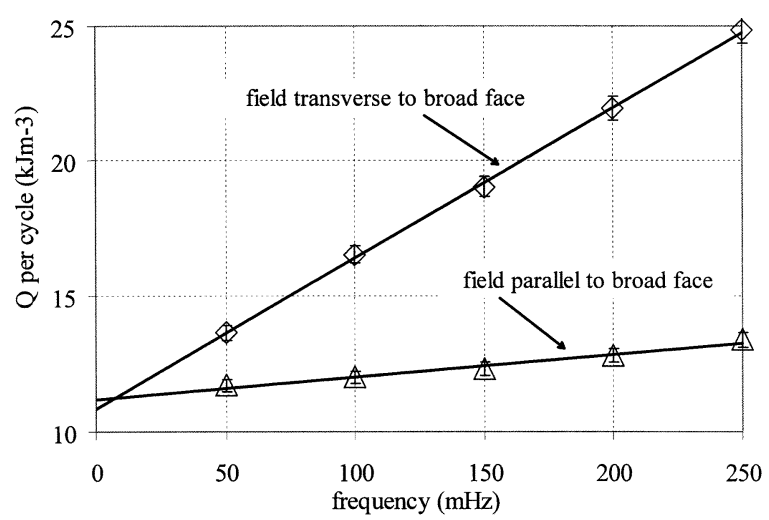

Fig. 5. Typical loss measurement on cable 003E.

Previous experience has shown us that the inter-strand contact resistance can vary greatly between different cool-downs of the same sample [10]. For this reason, we made $V-I$ measurements of $R_{a}$ on one length of the cable sample in situ during the same cool-down as the loss measurement. For cored cables however, $R_{c}$ is so high as to be invisible in the usual $V-I$ measurement and must be measured separately in a cut sample [11].

In order to separate the cable coupling loss from loss within the individual wires, the magnetization of a single wire with $13 \mathrm{~mm}$ twist pitch was measured at several different ramp rates. From this measurement we can derive the coupling and hysteresis losses in the wire.

\section{ANALYSIS OF THE LOSS MEASUREMENTS}

Our objective is to derive parameters and verify a model which can be used to predict losses in the synchrotron. We therefore start by using this model to predict the losses measured at Twente and then compare the results.

We start by using the wire magnetization measurements to derive two parameters:

a) $J_{c}$ as a function of $B$, which is then used to calculate hysteresis loss.

b) the effective transverse resistivity across the wire $\rho_{e t}$ which is used to calculate coupling loss within the wire.

Starting with $\mathrm{b}$ ), we plot the wire magnetization $M$ against $d B / d t$ at several different fields and find a straight line, as expected from:

$$
M_{e}=\frac{2 \dot{B} \tau_{f}}{\mu_{o}}
$$

where $\tau_{f}$ is the inter-filament coupling time constant

$$
\tau_{f}=\frac{\mu_{o}}{2 \rho_{e t}}\left\{\frac{p}{2 \pi}\right\}^{2}
$$

and $\rho_{e t}$ is the effective transverse resistivity across the wire, depending on the copper matrix, the interface resistance between $\mathrm{NbTi}$ and copper, and the geometry.

Fig. 6 shows a plot of our measured $\rho_{e t}$ as a function of $B$. Also shown for comparison is the magnetoresistance for copper [12] of similar RRR ( 220), scaled to fit at low field and showing a remarkably good agreement in variation with field.

By extrapolating the measured magnetization back to zero $\dot{B}$, we obtain the persistent magnetization of individual filaments, 


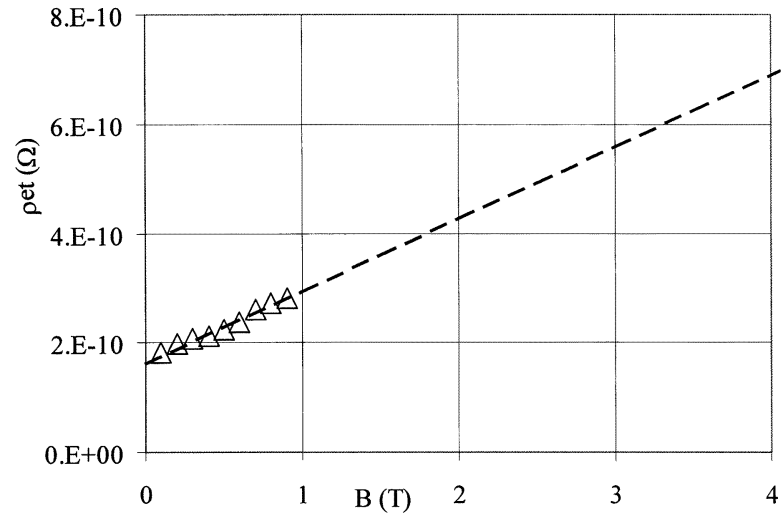

Fig. 6. Measured values of $\rho_{e t}$ for a wire of $13 \mathrm{~mm}$ twist pitch, dashed line shows magnetoresistance of copper, scaled to fit at low field.

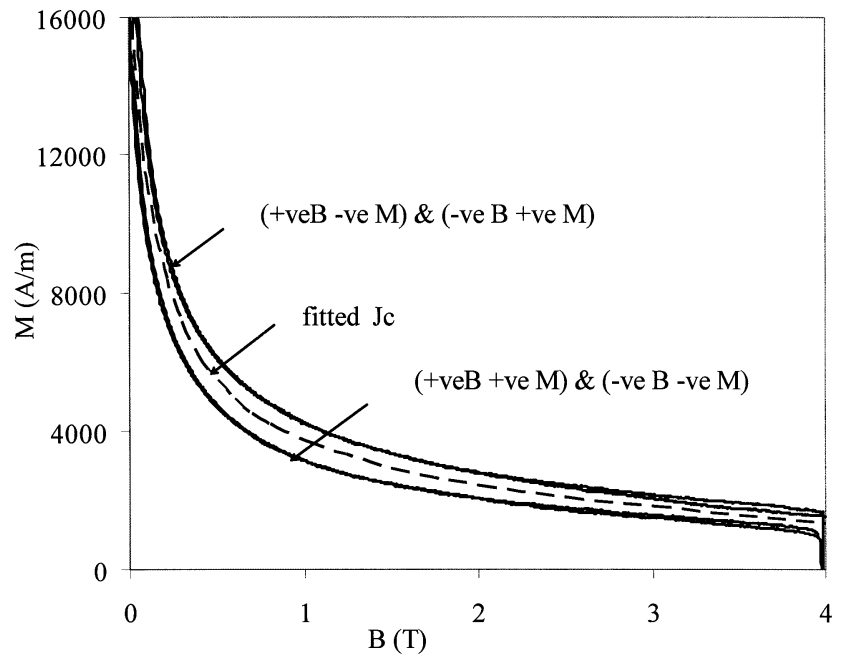

Fig. 7. Magnetization of a single wire extrapolated to zero $\dot{B}$ and plotted in the all positive quadrant; dashed line shows fitted $J_{c}$.

as plotted in Fig. 7, where the four quadrants of a symmetrical $\pm B$ magnetization loop have been plotted in the all positive quadrant.

The difference between "odd" and "even" quadrants is caused partly by field profile within the filaments, but mainly by reversible magnetization of the NbTi. To a good approximation, we may take a mean between them and calculate the bulk $J_{c}$ from:

$$
M_{f}=\frac{2}{3 \pi} \lambda_{f} J_{c} d_{f}
$$

where $\lambda_{f}$ is the filling factor and $d_{f}$ is the filament diameter. We fit the current density with a modified Kim Anderson approximation

$$
J_{c}=\frac{J_{o} B_{o}}{B+B_{o}}+A_{0}+A_{1} B .
$$

For the data of Fig. 6, we find:

$$
\begin{aligned}
& J_{o}=4 \times 10^{10} \mathrm{~A} \cdot \mathrm{m}^{-2} \\
& B_{o}=0.155 \mathrm{~T} \\
& A_{o}=4.8 \times 10^{9} \mathrm{~A} \cdot \mathrm{m}^{-2} \\
& A_{1}=-7.0 \times 10^{8} \mathrm{~A} \cdot \mathrm{m}^{-2} . \mathrm{T}^{-1}
\end{aligned}
$$

The methods of measuring $R_{c}$ and $R_{a}$ are described in [11] and these complete our list of parameters needed to calculate the losses. For a sinusoidal waveform of angular frequency $\omega$ and amplitude $\pm B_{a}$ we use the following formulae for loss per cycle:

a) Cable coupling via $R_{c}$ in transverse field

$$
Q_{t c}=\frac{\pi \omega}{120} \frac{B_{a}^{2}}{R_{c}} N(N-1) p \frac{c}{b} .
$$

b) Cable coupling via $R_{a}$ in transverse field

$$
Q_{t a}=\frac{\pi \omega}{24} \frac{B_{a}^{2}}{R_{a}}(N-1) \frac{p}{\operatorname{Cos}^{2} \theta} .
$$

c) Cable coupling via $R_{a}$ in parallel field

$$
Q_{p a}=\frac{\pi \omega}{32} \frac{B_{a}^{2}}{R_{a}}(N-1) \frac{b^{2}}{c^{2}} \frac{p}{\operatorname{Cos}^{2} \theta} .
$$

d) Inter filament coupling in the wires

$$
Q_{f}=\lambda_{w} 2 \pi \frac{B_{a}^{2}}{\mu_{o}} \omega \tau_{f} .
$$

e) Hysteresis in the filaments

$$
Q_{h}=\frac{8}{3 \pi} \lambda_{w} \lambda_{f} d_{f}\left\{J_{o} B_{o} \ln \left(\frac{B_{a}+B_{o}}{B_{o}}\right)+A_{o} B_{a}+A_{1} \frac{B_{a}^{2}}{2}\right\}
$$

where $\lambda_{w}$ and $\lambda_{f}$ are the filling factors of wire in the cable and NbTi filaments in the wire.

With these formulae and the measured $\rho_{e}, J_{c}, R_{a}$ and $R_{c}$, we calculate losses at $250 \mathrm{mHz}$ with a field amplitude $B_{a}=0.3 \mathrm{~T}$ as listed in Table III. For the filament coupling we take $\rho_{e t}=$ $1.9 \times 10^{-10} \Omega . \mathrm{m}$, as shown in Fig. 6, at the mean of the sinusoidal field, $B=0.15 \mathrm{~T}$. Of course, the measurements cannot distinguish between different types of coupling loss, so we also list the total calculated coupling loss for each field direction.

The $V-I$ measurements in [9] indicate that $R_{a}$ varies strongly across the cable, being much lower at the edges (Table III lists the average value). A simple calculation shows that if all the $R_{a}$ is located at the edge of the cable, the adjacent coupling in transverse field is increased by a factor 3 . For this reason, we include a line "total coupling transverse with edge Ra" in Table III, where the $Q_{t a}$ term has been increased to $3 \times Q_{t a}$.

Table IV compares the calculations with measurement. Firstly the hysteresis, which of course should not depend on field direction. It may be seen that the results are in reasonable agreement; deviations are probably caused by differences in $J_{c}$ between the cable and our measured wire. Secondly the transverse coupling, where the losses for cables B and $\mathrm{E}$ are nicely bracketed by the factor 3 edge enhancement in $R_{a}$. Cable $\mathrm{F}$ does not seem to show any edge enhancement, we don't know why.

In general however, we feel these calculations are in reasonable agreement with experiment and are good enough for outline design of the synchrotron. They do not suggest that the conventional theory is deficient or that we need to postulate additional current paths as suggested in [5].

\section{LOSSES IN THE SYNCHROTRON}

We now use this data to predict losses in a dipole of the synchrotron SIS200 [1] when operating under the ramping cycle 
TABLE III

CALCULATED LOSSES (J/CYCLE)

\begin{tabular}{|c|c|c|c|c|}
\hline \multicolumn{2}{|c|}{ cable } & 003B & $003 \mathrm{E}$ & $003 \mathrm{~F}$ \\
\hline crossover resistance & $R_{c}$ & $1.4 \mathrm{E}-02$ & $6.3 \mathrm{E}-02$ & $6.6 \mathrm{E}-04$ \\
\hline adjacent resistance & $\mathrm{R}_{\mathrm{a}}$ & $5.0 \mathrm{E}-05$ & $4.9 \mathrm{E}-06$ & 4.6E-06 \\
\hline wire twist pitch & $\mathrm{p}_{\mathrm{w}}$ & $4.0 \mathrm{E}-03$ & $6.0 \mathrm{E}-03$ & $6.0 \mathrm{E}-03$ \\
\hline transverse crossover loss & $Q_{\mathrm{tc}}$ & 144 & 32 & 3050 \\
\hline transverse adjacent loss & $Q_{\text {ta }}$ & 845 & 8628 & 9282 \\
\hline parallel adjacent loss & $Q_{p a}$ & 9 & 91 & 98 \\
\hline fil't coupling time const & $t_{f}$ & $1.3 \mathrm{E}-03$ & $2.9 \mathrm{E}-03$ & $2.9 \mathrm{E}-03$ \\
\hline filament coupling loss & $\mathrm{Q}_{\mathrm{f}}$ & 785 & 1767 & 1767 \\
\hline filament hysteresis & $Q_{h}$ & 10925 & 10925 & 10925 \\
\hline total coupling transverse & $Q_{t c}+Q_{t a}+Q_{f}$ & 1774 & 10427 & 14099 \\
\hline $\begin{array}{l}\text { total coupling transverse } \\
\text { with edge Ra }\end{array}$ & $\begin{array}{c}Q_{\mathrm{tc}}+3^{*} Q_{\mathrm{ta}} \\
+Q_{\mathrm{f}}\end{array}$ & 3464 & 27684 & 32662 \\
\hline total coupling parallel & $Q_{p a}+Q_{f}$ & 794 & 1858 & 1865 \\
\hline
\end{tabular}

TABLE IV

EXPERIMENTAL LOSSES COMPARED WITH CALCULATION (J/CYCLE)

\begin{tabular}{cccc}
\hline \multicolumn{1}{c}{ cable } & $003 \mathrm{~B}$ & $003 \mathrm{E}$ & $003 \mathrm{~F}$ \\
\hline hysteresis transverse & 10230 & 10849 & 11350 \\
hysteresis transverse exp/theo & 0.94 & 0.99 & 1.04 \\
hysteresis parallel & 10073 & 11201 & 11683 \\
hysteresis parallel exp/theo & 0.92 & 1.03 & 1.07 \\
coupling transverse & 2974 & 13915 & 12460 \\
coupling transverse exp/theo & 1.68 & 1.33 & 0.88 \\
coupling trans + edge Ra exp/theo & 0.86 & 0.50 & 0.38 \\
coupling parallel & 1008 & 2070 & 1082 \\
coupling parallel exp/theo & 1.27 & 1.11 & 0.58 \\
\hline
\end{tabular}

TABLE V

LOSSES CALCULATED FOR THE SYNCHROTRON

\begin{tabular}{|c|c|c|c|c|c|}
\hline \multicolumn{2}{|c|}{ cable } & 003B & $003 \mathrm{~B} 3.5$ & 003F & $002 N C$ \\
\hline crossover $R_{c}$ & $\mu \Omega$ & $1.4 \mathrm{E}-02$ & $1.4 \mathrm{E}-02$ & $6.6 \mathrm{E}-04$ & $5.0 \mathrm{E}-05$ \\
\hline adjacent $R_{a}$ & $\mu \Omega$ & $5.0 \mathrm{E}-05$ & $5.0 \mathrm{E}-05$ & $4.6 \mathrm{E}-06$ & $5.0 \mathrm{E}-05$ \\
\hline edge factor & & 2.5 & 2.5 & 1.0 & 1.0 \\
\hline filament diameter $d_{f}$ & $\mu \mathrm{m}$ & $6.0 \mathrm{E}-06$ & $3.5 \mathrm{E}-06$ & $6.0 \mathrm{E}-06$ & $6.0 \mathrm{E}-06$ \\
\hline amping power $\mathrm{Pr}$ & Watt & 13.1 & 9.9 & 24.3 & 47.4 \\
\hline average power $\mathrm{P}_{\mathrm{av}}$ & Watt & 7.7 & 5.9 & 14.3 & 28.0 \\
\hline \multicolumn{6}{|c|}{ loss components as a fraction of the total } \\
\hline \multirow{5}{*}{\multicolumn{2}{|c|}{$\begin{array}{l}\text { transverse crossover } \mathrm{P}_{\mathrm{tc}} \\
\text { transverse adjacent } \mathrm{P}_{\mathrm{ta}} \\
\text { parallel adjacent } \mathrm{P}_{\mathrm{pa}} \\
\text { filament coupling } \mathrm{P}_{\mathrm{f}} \\
\text { hysteresis } \mathrm{P}_{\mathrm{h}}\end{array}$}} & $1.0 \%$ & $1.3 \%$ & $11.2 \%$ & $75.7 \%$ \\
\hline & & $18.8 \%$ & $24.8 \%$ & $44.3 \%$ & $2.1 \%$ \\
\hline & & $0.2 \%$ & $0.3 \%$ & $1.3 \%$ & $0.1 \%$ \\
\hline & & $12.4 \%$ & $16.4 \%$ & $6.7 \%$ & $3.4 \%$ \\
\hline & & $67.6 \%$ & $57.3 \%$ & $36.5 \%$ & $18.7 \%$ \\
\hline
\end{tabular}

described in the introduction, using the method of calculation described in [10]. The dipole is $2.6 \mathrm{~m}$ long and the cables are based on the samples tested, but with a reduced copper/NbTi ratio of $1.8: 1$ and a wire twist pitch of $4 \mathrm{~mm}$. Loss power is calculated during ramping and also averaged over the whole cycle. To allow for low $R_{a}$ on the cable edge, the loss $Q_{t a}$ is multiplied by a factor which just brings the theory into line with the measurements in Table IV. Table V presents the results, starting with our lowest loss cable 003B. It may be seen that hysteresis is by far the largest component of loss. We therefore include a second column, having the 003B cable parameters, but with $3.5 \mu \mathrm{m}$ filaments, chosen as our estimate of the smallest diameter possible without incurring too much proximity coupling in a pure copper matrix. In fact the 003B 3.5 column includes an estimate of proximity coupling, based on [13], which adds $\sim 10 \%$ to the hysteresis loss.

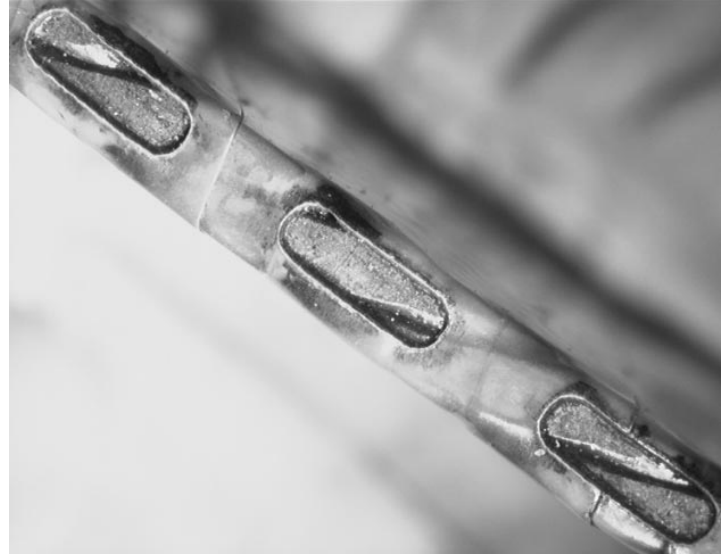

Fig. 8. Cooling slots cut by laser on the inner edge of the cable.

TABLE VI

CAlculated TEMPERATURe Rises

\begin{tabular}{lccc}
\hline & \multicolumn{1}{c}{ cable } & 003B & 003F \\
\hline$\Delta \theta$ cable & $\mathrm{mK}$ & 9 & 8 \\
$\Delta \theta$ heat transfer & $\mathrm{mK}$ & 70 & 128 \\
$\Delta \theta$ total & $\mathrm{mK}$ & 79 & 136 \\
\hline
\end{tabular}

If ramp rate quenching turns out to be a problem, it may be necessary to use a lower inter-strand resistance, so the third column is based on cable 003F. It may be seen that the lower $R_{a}$ and $R_{c}$ have roughly doubled the total loss.

Finally, the last column is included with the same $R_{a}$ as 003B, but with $R_{a}=R_{c}$ to represent a cable with no core. It may be seen that this has increased the loss substantially. Of course, if an inter-strand resistance similar to $003 \mathrm{~F}$ turns out to be necessary, the no-core loss would be extremely high.

The losses in Table $\mathrm{V}$ are somewhat higher than presented in [10] because our measured $\rho_{e t}$ is lower than earlier estimates, the matrix ratio is reduced to $1.8: 1$ and $J_{c}$ is higher.

\section{TEMPERATURE RISE AND COOLING}

For good cooling during the ramp, we have developed a new insulation with cooling holes cut by laser in the Kapton wrap along the inner edge of the cable, as shown in Fig. 8.

Trial sections of coil have been made from cable with this insulation and found to have an inter-turn voltage breakdown of $\sim 1.5 \mathrm{kV}$, giving a comfortable safety margin against the expected ramping and quench voltages in the dipole.

Cooling in the dipole will be provided by forced-flow supercritical helium along the inner surface of the winding, so the temperature rise in the cable has two components:

a) conduction across the width of the cable.

b) heat transfer to the helium.

Heat is conducted across the cable in two ways:

i) via the thermal resistance between adjacent wires.

ii) along the wires in a diagonal direction.

We estimate i) from $R_{a}$ assuming the Wiedemann-Franz Law: a reasonable guess but not, to our knowledge, verified for contact resistances. Heat transfer is estimated via a Dittus-Boelter correlation for supercritical helium [14], assuming the coolant to be at $4.4 \mathrm{~K}$ with a pressure of $5 \mathrm{~atm}$. and flow rate of $100 \mathrm{gm} / \mathrm{sec}$. Table VI summarizes the temperature 
rises a) and b) plus the total, for an insulation in which the slots expose $26 \%$ of the cable inner edge. It may be seen that temperature drops across the cable are negligible and that, even with such a small cooling area, the heat transfer temperature rises are small.

With no cooling slots at all, the temperature drop across the Kapton would be $\sim 0.2 \mathrm{~K}$. Much more serious however is the likely prospect of a static gas film between the Kapton and cable, in which case the temperature drop would be $\sim 1.5 \mathrm{~K}$-far too near the allowable temperature margin for any practical accelerator.

\section{CONCLUSION}

Cored Rutherford cables are the preferred choice for the proposed GSI fast pulsed synchrotron because they have reasonable losses, while retaining a low inter-strand resistance.

We have shown that the measured losses of three different cored cables can be explained reasonably well in terms of conventional theory. Because of the variability of inter-strand resistance, it is important to measure it in situ, during the same cool-down as the loss measurement. There seems to be some enhancement of loss in transverse field caused by the adjacent resistance $R_{a}$ being lower at the cable edges.

Extrapolating these measured values to the planned synchrotron we find acceptable levels of loss. Using our new insulation with cooling holes, we calculate temperature rises in the cable which will not significantly degrade its performance.

There are good reasons to believe that a low inter-strand resistance in the cable will be helpful in reduce the sensitivity of quench current to high ramp rates, but this remains conjectural at present. It can only be verified by testing prototype magnets, due to start later this year.

\section{ACKNOWLEDGMENT}

The authors are grateful to G. Staupendahl and colleagues at the Technical University of Jena for developing techniques for producing cooling holes in the insulation and to A. P. Verweij at CERN for help in interpreting the $V-I$ data on inter-strand resistance.

\section{REFERENCES}

[1] G. Moritz, "Superconducting magnets for the "international accelerator facility for beams of ions and antiprotons' at GSI," in ASC Conf. 2002, Paper 4LA01.

[2] A. D. Kovalenko, N. N. Agapov, A. M. Donyagin, H. G. Khodzhibagiyan, G. L. Kuznetsov, G. Moritz, G. Hess, J. Kaugerts, E. Fischer, and C. Muehle, "Superferric model dipole magnet with the iron yoke at $80 \mathrm{~K}$ for the GSI future fast cycling synchrotron," in ASC Conf. 2002, Paper 4LA02.

[3] M. Anerella, J. Cozzolino, J. Escallier, G. Ganetis, A. Ghosh, R. Gupta, M. Harrison, A. Jain, S. Kahn, E. Killian, W. Louie, A. Marone, J. Muratore, S. Plate, M. Rehak, W. Sampson, J. Schmalzle, R. Thomas, P. Thompson, P. Wanderer, and E. Willen. The RHIC magnet system. Nucl. Inst. Meth. [Online]. Available: http://www.bnl.gov/magnets/publications/index.asp, to be published.

[4] D. Wake, D. Gross, R. Yamada, and B. Blatchley, IEEE Trans. Magn., vol. MAG-15, no. 1, p. 141, 1979.

[5] M. D. Sumption, E. W. Collings, R. M. Scanlan, S. W. Kim, M. Wake, T. Shintomi, A. Nijhuis, and H. J. ten Kate, "AC loss in cored stabrite cables in response to external compaction and variation in core thickness and width," Cryogenics, vol. 41, pp. 733-744, 2001.

[6] A. K. Ghosh, W. B. Sampson, and M. N. Wilson, "Minimum quench energies of rutherford cables and single wires," IEEE Trans. Appl. Superconduct., vol. 7, p. 954, June 1997.

[7] A. Devred and T. Ogitsu, "Ramp rate sensitivity of SSC magnet prototypes," in Frontiers of Accelerator Technology, S. I. Kurokawa, M. Month, and S. Turner, Eds, Singapore: World Scientific, 1996, pp. 184-308.

[8] H. Bruck et al., "Observation of periodic patterns in the persistent current fields of the superconducting HERA magnets," in Proc. IEEE Particle Accelerator Conference, San Francisco, CA, 1991, p. 2149.

[9] H. Hemmes, M. J. Woudstra, H. J. ten Kate, and J. Tenbrink, "Transport current dependence of the hysteresis loss in silver-sheathed BSCCO-2212 conductors," Cryogenics, vol. 34, Suppl. ICEC, pp. 567-570, 1994.

[10] M. N. Wilson, G. Moritz, M. Anerella, G. Ganetis, A. K. Ghosh, W. V. Hassenzahl, A. Jain, P. Joshi, J. Kaugerts, C. Muehle, J. Muratore, R. Thomas, G. Walter, and P. Wanderer, "Design studies on superconducting $\operatorname{Cos} \theta$ magnets for a fast pulsed synchrotron," IEEE Trans. Applied Superconduct., vol. 12, p. 313, Mar. 2002.

[11] M. Anerella, A. K. Ghosh, R. Soika, P. Wanderer, M. N. Wilson, W. V. Hassenzahl, J. Kaugerts, and G. Moritz, "Inter-strand resistance measurements in cored Rutherford cables," in ASC Conf. 2002, Paper 5LG02.

[12] F. R. Fickett, "Oxygen free high conductivity copper at 4K: Resistance and magnetoresistance," IEEE Trans. Magn., vol. MAG-19, no. 3, p. $228,1983$.

[13] A. K. Ghosh, W. B. Sampson, E. Gregory, and T. S. Kreilick, "Anomalous low field magnetization in fine filament NbTi conductors," IEEE Trans. Magn., vol. MAG-23, no. 2, p. 1724, 1987.

[14] V. Arp, "Force flow single phase helium cooling systems," Adv. Cryo. Eng., vol. 17, p. 343, 1972. 